\title{
Defect Detection in Fabric using Wavelet Transform and Genetic Algorithm
}

\author{
Depavath Harinath ${ }^{1}$, K. Ramesh Babu ${ }^{2}$,P.Satyanarayana ${ }^{3}$, M.V. Ramana Murthy ${ }^{3}$ \\ ${ }_{1}^{1}$ Dept. of Computer Science, HRD Degree and P.G College Narayanaguda, Hyderabad, Telangana, India. \\ ${ }^{2}$ Dept. of Mathematics, M.V.S.R Engineering College, Nadergul, R.R.District, Hyderabad, Telangana, \\ India. \\ ${ }^{3}$ Dept. of Computer Science, Osmania University, Hyderabad,Telangana, India. \\ harinath.depavath@gmail.com
}

\begin{abstract}
Fabric defect detection is one of the indispensable units in the manufacturing industry to maintain the quality of the end product. Wavelet transform is well suited for quality inspection application due to its multi-resolution representation and to extract fabric features. In this paper a new scheme is proposed for fabric defect detection in textile industry. For this purpose, all coefficients were extracted from perfect fabric. These coefficients can defect main fabric image $\&$ indicate defects of fabric textile by optimal subset of these coefficients. For finding defects a suitable subset of Genetic Algorithm is used in this process. The Shannon entropy is used as evaluation function in Genetic Algorithm By using two separable sets of wavelet coefficients for horizontal and vertical defects, it was seen that we get better results for defect detection. The advantage of this approach is that it improves accuracy of fabric defect detection as well decreases computation time.
\end{abstract}

Keywords - Wavelet transform, Genetic Algorithm, Fabric Defect Detection.

\section{Introduction}

In textile industry, quality control is very important as it directly affects the final product of the industry. Hence to improve quality of textile industry, inspection of textile is very important. In ancient period the inspections in textile industry were done manually which took long time for detection \& it become highly expensive due to labor costing. But now inspections are done with the help of automatic inspector machineries. Failure in selection of textile directly affects on quality of production in textile industry \& manual inspection requires large time, hence it increases time for manufacturing of product. To improve inspection, modern computer vision inspection system came into existence. It improves accuracy of finding defects and also reduces labor power.

There are numerous reports that shows computer vision based inspection has become very effective in detecting defects in textile industry [1]. Automatic inspections are based on computer vision method \& they inspect the defects automatically. Defect in textile industry can be detected by various methods. Research areas in detecting defects are textile, surface of wood, tile, aluminum, distance difference, etc [2]. Uniform, random or patterned textures are the various areas for detecting defects in textile [3]. In this proposed method, fabric defect detection is done by distance difference. In this 
proposed system, wavelet transformer is used as filter \& Genetic algorithm as defect detector. It improves accuracy as well reduces computational time.

A fabric defect is any abnormality in the fabric that hinders its acceptability by the consumer. The textile processing does not eliminate variability incurred during different steps in textile manufacturing. As materials flow from one stage of processing to another, components of variability are added and the final product may involve a cumulative variability that is much higher than the variability of the input fibers and thus it cause a defect in the fabric. The main factors that lead to fabric defects are failure of opening and cleaning the machines that completely eliminate contaminants and trash particles, and it may leads to spinning, weaving and knitting related defects. So the fabric inspection has to identify all types of defects with minimum effort.

Several methods have been proposed to address the problem of detecting defects in textile fabrics, including statistical, spectral and model based approaches.

The wavelet transform provides a solid and unified mathematical framework for the analysis and characterization of an image at different scales. It provides both time and frequency information, and can be successfully applied for textile defect detection. Fabric defect detection based on wavelet transform performs better with less computation than the traditional statistical texture analysis approaches in identifying defects.

The flow of proposed methods is explained in three main parts. First part consists of extracting coefficient through wavelet transform \& obtaining coefficient of subset. In second part the suitable subsets are selected through genetic algorithm. Finally in third part suitable subsets are applied to other images and then defects are detected through genetic algorithm.

In first part, the proposed system uses wavelet filter for extracting coefficient of subset. This filter puts special information in each quarter of filtered image. The initial image is divided into four parts by two dimensional wavelet transform. The first part is an approximation of initial image and the other parts include components with high frequencies \& low frequencies. By using wavelet transform as filter the wavelet coefficients are obtained. Then genetic algorithm is applied to find suitable subset. Then defects are obtained by fabric defect detection.

\section{Literature Survey}

Wavelet Transform is decomposition of a signal into constituent parts. This decomposition can done in many ways,

1. Fourier series: Harmonic sinusoids; Single integer index.

2. Fourier transform: Non- harmonic sinusoids; single real index.

3. Walsh Decomposition: "harmonic" square waves; single integer index.

4. Kahunen - Loeve decomposition: Eigen function of covariance; single real index.

5. Short - Time FT (STFT): windowed, non-harmonic sinusoids; double index.

Wavelet transform provide waves in frequency \& time domain. Duration of decomposition of signal is limited \& has limited band-pass components. High frequency components have short duration \& have wide band. Whereas, low frequency components have longer duration \& have narrow band. 
The wavelet transform is used as filter in this proposed system. The wavelet transform uses a family of wavelet functions and its associated scaling function is used for decomposition of original image into different sub-bands, providing both frequency \& spatial function.

Wavelet transform analyses the signal by a group of orthogonal functions in the form of $\Psi \mathrm{m}, \mathrm{n}(\mathrm{X})$, that is computed from translation $\&$ dilation of the mother wavelet $\Psi$. These orthogonal functions are shown below,

$$
\Psi_{\mathrm{m}, \mathrm{n}}(\mathrm{x})=2 \frac{-m}{2} \Psi\left(2^{-\mathrm{m}} \mathrm{x}^{-\mathrm{n}}\right)
$$

$m$ and $n$ are real numbers in this equation.

Equation $2 \& 3$ are used as analysis \& synthesis formulas respectively.

$$
\begin{gathered}
\mathrm{C}_{\mathrm{m}, \mathrm{n}}=\int_{-\infty}^{+\infty} f(x) \psi_{\mathrm{m}, \mathrm{n}} \mathrm{n}(\mathrm{x}) \mathrm{dx} \\
f(\mathrm{x})=\sum_{\mathrm{m}, \mathrm{n}} \mathrm{C}_{\mathrm{m}, \mathrm{n}} \Psi_{\mathrm{m}, \mathrm{n}}(\mathrm{x})
\end{gathered}
$$

Coefficients of this transform are obtained recursively. A synthesis of $j$ is shown in equation 4.

$$
f(x)=\sum{ }_{k} C_{0, k} \Phi_{0, k}(x)=\sum \mathrm{kCj}_{+1}, \mathrm{k}^{\Phi} \mathrm{j}_{-\mathrm{D}} \mathrm{d}_{\mathrm{j}+1, \mathrm{k}} \Psi_{\mathrm{j}+1, \mathrm{k}}(\mathrm{x})
$$

Two dimensional wavelet transform is used as filter.

The two dimensional wavelet transform divides image into four part .Other parts include components with high frequencies of image \& edge points' shows vertical, horizontal \& diagonal direction. The wavelet transform uses two types of filter, high frequency filter \& low frequency filter. When first order of DWT is applied on image, the image is divided into four part is $I_{H H}, I_{L L}, I_{L H} \& I_{H L}$. Where, $H, L$ is the high \& low bound filter respectively

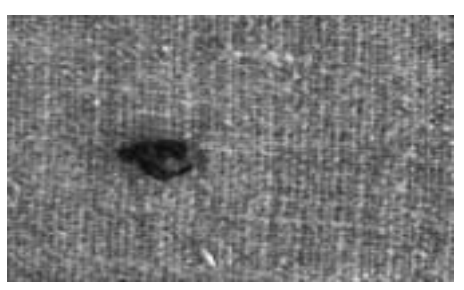

Figure (1): Original image.

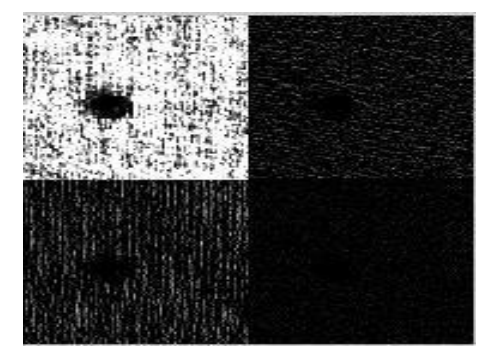

URL:http://dx.doi.org/10.14738/tmlai.36.1551 
Figure (2): Wavelet Decomposed image after first order

Wavelet transform evaluates the difference in information in two different resolutions \& creates a multi-resolution view of image [2]. Hence wavelet transform is used for fabric defect detection. After decomposing image by wavelet filter, the proposed system gets coefficients of subset. For finding suitable subset the proposed system uses the genetic algorithm.

\section{The Genetic Algorithm}

In a genetic algorithm, a population of candidate solutions to an optimization problem is evolved towards better solutions [4]. The genetic algorithm is iterative process, the evolution starts from a population of randomly generated individual, with the population in iteration is called a generation.

In each generation the fitness function of every individual is calculated. The fitness function is usually the value of objective function which optimizes the better solution to the problem. The fit individuals are selected from the current population $\&$ each genome of individual is modified for formation of next generation. In next iteration, new generation of candidate solutions is used in next iteration of the algorithm. The algorithm is repeated until satisfactory fitness level is not reached. The generation of second population is done through the genetic operation such as crossover \& mutation.

The simplest form of genetic algorithm involves three types of operators [13]:

1. Selection: This operator selects chromo-somes from population for reproduction. It fitter the chromosome more times so that it is likely to be selected to reproduce.

1. Crossover: The operator in which the subsequences of two chromosomes are exchanged to create new child is called as crossover. This operator roughly mimics biological recombination between two single chromosome organisms.

2. Mutation: This operator randomly flips some bits in a chromosome. Mutations occur at each bit position in a string with some probability.

The Genetic algorithm work as follows [13]

1. Start with a randomly generated population of N L-bit chromosome.

2. Calculate the fitness function $F(x)$ of each chromosome $x$ in the population.

3. Repeat the following steps (a)-(c) until $\mathrm{N}$ offspring have been created:

a) Select a pair of parent chromosome from the current population, with the probability of selection being an increasing function of fitness. Selection is done with replacement," meaning that the same chromosome can be selected more than once to become parents."

b) With probability Pc (the crossover probability) is used to form two offspring. If no crossover takes place, form two offspring that are exact copies of their respective parents.

c) Mutate the two offspring at each locus with probability Pm (the mutation probability), and pace the resulting chromosomes in the new population.

4. Replace the current population with the new population.

5. If the stopping condition has not satisfied, go to step 2 . 


\section{Fabric Defect Detection}

In second part, the propose method obtains suitable subset through genetic algorithm after obtaining coefficients of subset by using wavelet transform. To obtain defect in fabric the proposed system has to apply the suitable subset to the images. This suitable subset detects the main image of fabric \& indicates the defects in the fabric image. If the optimization problem in Equation 5 can be solved, then suitable subset of wavelet coefficient can be

$$
\operatorname{Min} J=C^{*} P^{*} P^{\top *} C^{\top}
$$

Where,

$$
\mathrm{C}_{\mathrm{j}(\mathrm{e})}=\sum_{k=0}^{n-1} C_{\mathrm{k}} C_{\mathrm{k}-2 \mathrm{~J}}=0
$$

In this equation $\mathrm{J}$ is the normal form in high frequency components of image, $\mathrm{n}$ is the number of coefficients, $C$ is subset of wavelet coefficient as a solution, $p$ is an array of image's pixel values. To minimize J function through c subset, the proposed system uses the genetic algorithm. In this algorithm, each solution $\mathrm{c}$ is chromosome $\&$ different function can be calculated. As the proposed system uses the wavelet transform as filter, this filter divides image into vertical \& horizontal directions. Due this it does not give good result when fabric is not symmetric. So, to obtain better result, two separate filter should be used(horizontal \& vertical filter).If $W 1, W 2$ is horizontal \& vertical filers \& $u$ is a perfect image, then filtered image $F$ can be obtained by,

$$
\mathrm{F}=\mathrm{W} 1 * \mathrm{U} * \mathrm{~W}_{2}^{\top}
$$

In this proposed system the fitness function for filtered image $F$ is calculated by using sum of squares $\&$ entropy functions. Eq. $7 \& 8$ shows these functions respectively.

$$
\operatorname{Sum}=\sum_{i=1}^{n} \sum_{j=1}^{m} F(i, j)^{2}
$$

Where, $n=$ number of lines, $m=$ column in images. \& $F(i, j)$ is the pixel value of the image, in line umi \& column j.

$$
\mathrm{H}=-\sum_{l=1}^{n} d(j) * \log _{2}(\mathrm{~d}(\mathrm{j}))
$$

Where,

$\mathrm{n}=$ number of grey levels, $\& \mathrm{~d}(\mathrm{i})$ is probability of the grey levels in $\mathrm{i}$ in the filtered image $\mathrm{F}$. This probability is the real number between 0 \& 1. With the help of Eq.7 \& 8, optimization problem can be obtained by minimizing J function in Eq. $9 \& 10$ resp.

$$
\begin{gathered}
\mathrm{J}=\sum_{l=1}^{n} \sum_{j=1}^{m}\left(\mathrm{~W} 1 * \mathrm{U} * \mathrm{~W}_{2}^{\mathrm{T}}\right)^{2} \\
\mathrm{~J}=\operatorname{Entropy}\left(\mathrm{W}_{1} * \mathrm{U} * \mathrm{~W}_{2}{ }^{\mathrm{T}}\right)
\end{gathered}
$$

The wavelet coefficients are obtained on monotony background of entropy function. So, if the entropy function is very small, the image will be more monotonic. Whereas, sum of the square function of image is optimized by minimizing its pixel values. Entropy has simpler computation as compare to sum of squares. So, in this proposed system, entropy function is used for calculation of fitness function of Genetic Algorithm. 


\section{Expected Result and Conclusion}

In this proposed system, the database of fabric is used for finding defect in it. First, the grey image is converted into contrast enhanced image. After conversion the histogram of image is obtained. This histogram is compared with the histogram of the processed image. After comparison, the wavelet transform is used as filter. In this session, the two dimensional wavelet filter is used. This filter divides the image into four parts \& the coefficients of subsets are extracted through wavelet filter.

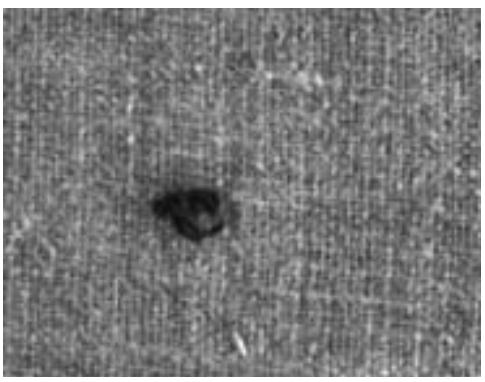

Figure (3): Original grey image

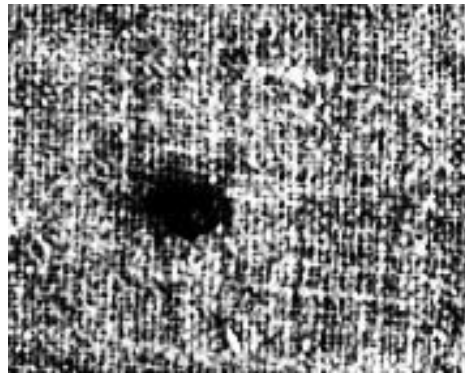

Figure (4): Contrast Enhanced image

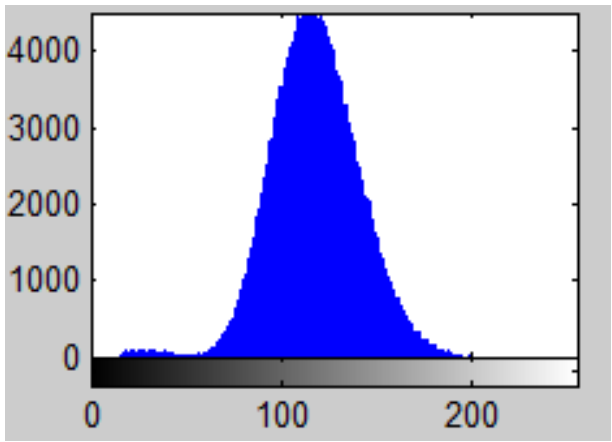

Figure (5): Histogram of original image

After comprising the histogram with processed image, wavelet decomposition is performed. 


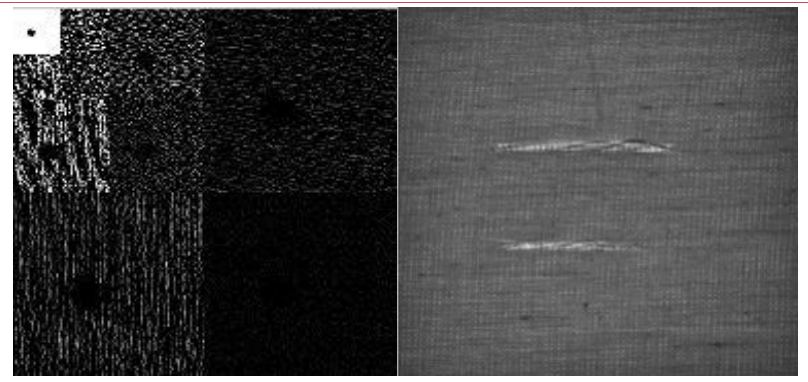

Figure (6): Wavelet Decompose image

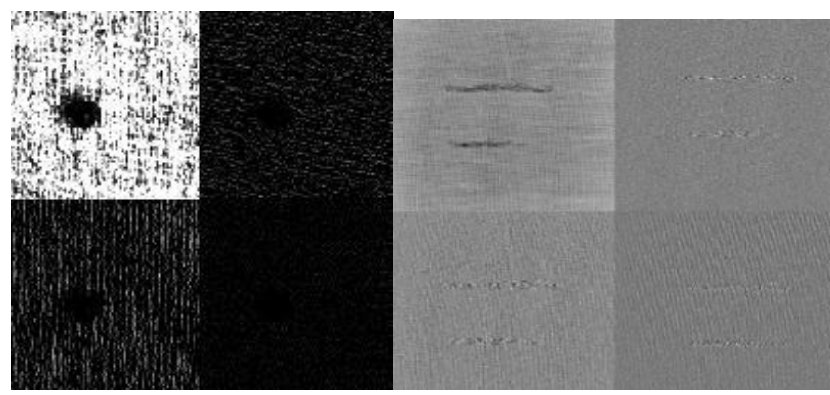

Figure (7): Decomposed image after first stage

After that the proposed system uses genetic algorithm to find suitable subset, then this suitable subset is applied to other images. Original image is deleted \& the defects are gained.

\section{REFERENCES}

[1] Mahajan P. M., Kolhe S. R and Patil P.M“A review of automated fabric defect detection”.

[2] Narges Heidari, Reza Azmi \& Boshra Pishgoo "Fabric Textile Defect Detection, By Selecting A Suitable Subset of Wavelet Coefficients, through Genetic Algorithm", International Journal of Image Processing (IJIP), Volume (5): Issue (1): 2011

[3] Lijing Wang, Zhongmin Deng and Xungai Wang "Application of Wavelet Transform Method for Textile Material Feature Extraction".

[4] B.V. Ramana Reddy1, A. Suresh2, M. Radhika Mani3, and V.Vijaya Kumar4 "Classification of Textures Based on Features Extracted from Preprocessing Images on Random Windows", International Journal of Advanced Science and Technology Volume 9, August, 2009.

[5] www.wikipedia.com

[6] Gonzalez R C \& Woods R E, Digital Image Processing, 1982,194.

[7] Image Processing Tool Box for use with Matlab (The Math Works Inc. USA), 2005. 
[8] Duda,"Pattern Classification."Advances in Computational Research, ISSN: 0975-3273, Volume 1, Issue 2, 2009, pp-18-29.

[9] Mahajan P.M.1, Kolhe S.R.2 and Patil P.M.3"A review of automatic fabric defect detection techniques" Bioinfo Publications, Advances in Computational Research, ISSN: 0975-3273, Volume 1, Issue 2, 2009.

[10] Xianghua Xie"A Review of Recent Advances in Surface Defect Detection using Texture analysis Techniques" Electronic Letters on Computer Vision and Image Analysis 7(3):1-22, 2008

[11] K.Srinivasan and P.H. Dastor and P. Radhakrishnaihan, and S. Jayaraman "FDAS: A Knowledgebased Frame Detection Work for Analysis of Defects in Woven TextilStructures", Journal of Textile Institute, vol. 83, pp. 431-447, (1992).

[12] R. Chin, "Automated Visual Inspection Techniques and Applications: A Bibliography", Pattern Recognition, 15(4): pp. 343-357, (1982).

[13] Z. Guanng and W. Jianxia, "Fabric Defect Detection Method Based on Image Distance Difference", Electronic Measurement and Instruments, (2007), pp. 822 -825.

[14] D. Chetverikov and A. Hanbury, "Finding Defects in Texture Using Regularity and Local Orientation." Pattern Recognition. 35(10), pp. 2165-2180, (2002).

[15] X.Z. Yang and G. Pang and N. Yung, "Discriminative Fabric Defect Detection Using Adaptive Wavelets", Optical Engineering, pp. 3116-3126, December (2002).

[16] X.Z. Yang and G. Pang and N. Yung, "Robust Fabric Defect Detection and Classification Using Multiple Adaptive Wavelets", Image and Signal Processing, IEEE Proceedings, volume 152, PP. 712-723, (2005)

[17] D. Chetverikov " Measuring the Degree of Texture Regularity", In Proceedings of International Conference on Pattern Recognition, 1984, volume 1, PP. 80-82, (1984).

[18] Yu.zhang, zhaoyang Lu, Jing Li,"Fabric Defect Detection \& classification using Gabor filters \& Gaussian Mixture Model", Springer-LNCS, pp: 635-644,(2010).

[19] HAN leil, LI zhong,"quick defect detection based on structure character of woven fabric image \& wavelet transform", computer engineering \& design",(2009)

[20] Rong Fu, Meihong Shi, Hongli Wei, Huijuan chen,"fabric defect detection based on adaptive local binary patterns", international conference on robotics \& bi omimetics, pp: 1336-1340,(2009)

[21] S.Mallat, "A Wavelet Tour of Signal Processing", Academic Press, 2nd ed., San Diego,(1999). 
[22] S.Mallat, "A Theory for Multiresolution Signal Decomposition: the Wavelet Representation", IEEE Transactions on Pattern Anal. and Mach. Intell., vol. 11, no. 7, pp. 674-693, (1989)

[23] Z. Michalewicz, "Genetic Algorithms + Data Structures =Evolution Programs", Al Series.Springer-Verlag, New York, 3rd edition, (1996)

[24] M. Mitchell, "Genetic Algorithm: An Overview", pp: 31-39, (1995).

[25] L.W. Leung and B. King an, "Comparison of Image Data Fusion Techniques Using Entropy and INI". 22nd Asian Conference on Remote Sensing, November (2001)

[26] Workgroup on "Texture Analysis of DFG, "TILDA Textile Texture Database", http://Imb.informatik.uni-freiburg.de/ research/dfg-texture/tildeNarges Heidari, Reza Azmi \& Boshra Pishgoo.

[27] P.Brodatz, Textures: A Photographic Album for Artists and Designers, Dover, New York, (1966).

[28] Depavath Harinath, "Enhancing Security through Steganography by using Wavelet Transformation and Encryption" in International Journal of Modern Trends in Engineering and Research (IJMTER) Volume 02, Issue 08, [August- 2015] 